\title{
Một vòng thư viện, cuối tháng 8-2021
}

\author{
Nguyễn Thanh Thanh Huyền
}

Hà Nội, ngày 24 tháng 8 năm 2021

Đầu tháng 8, cuốn monograph tiêu đề $A$ Mindsponge-Based Investigation into the Psycho-Religious Mechanism Behind Suicide Attacks [1] xuất bản bởi Walter de Gruyter GmbH, 2021 (De Gruyter Poland / Imprint: Sciendo; ISBN: 9788366675582).

Tới nay, đã phát hành được 3 tuần, và một số hệ thống bán lẻ lớn đã có sẵn trong kho (Amazon $F R, D E$ ), hoặc có thể đặt sách trước (Amazon US https://www.amazon.com/dp/8366675580/, UK, JP, hay Barnes\&Noble, https://www.barnesandnoble.com/w/1139867936).

Một số hệ thống khu vực cũng đã có thể đặt sách, như Kinokunya ở Nhật (https://www.kinokuniya.co.jp/f/dsg-02-9788366675582) hay Blackwell's ở Anh (https://blackwells.co.uk/bookshop/product/9788366675582)

Bên cạnh đó, còn một thông tin đáng theo dõi là việc yết sách trên các thư viện quốc tế. Hiện nay, một hệ thống trợ giúp việc tìm kiếm thư viện nơi lưu trữ sách chính là WorldCat OCLC. Ít nhất có hai địa chỉ lưu trữ sách trên hệ thống OCLC là:

- http://www.worldcat.org/oclc/1261727631

- http://www.worldcat.org/oclc/1264263862

Qua đó có thể thấy được những thư viện quốc tế sau đây có lưu trữ:

1. Bibliothek der Brandenburgische Technische Universität Cottbus-Senftenberg. UB Cottbus. Cottbus, 03044 Germany. https://b-tu.kobv.de/redirect.do?target=1103753\&type=opac\&plv=2

2. Universitätsbibliothek Passau. Passau, 94032 Germany. https://literatursuche.ub.unipassau.de/TouchPoint/perma.do?q=+1035\%3D\%22BV047416805\%22+IN+\%5B2\%5D\&v=upa\&I= de

3. Hochschule Augsburg, Bibliothek. Augsburg, 86161 Germany. https://infoguide.hsaugsburg.de/TouchPoint/perma.do? $q=+1035 \% 3 \mathrm{D} \% 22 \mathrm{BV} 047416805 \% 22+\mid \mathrm{N}+\% 5 \mathrm{~B} 2 \% 5 \mathrm{D} \& \mathrm{v}=$ fha\& $=$ de

4. Hochschule Coburg, Zentralbibliothek. Coburg, 96450 Germany.

5. Hochschule Landshut, Hochschule für Angewandte Wissenschaften, Bibliothek

6. Univeristy of Applied Sciences Landshut. Landshut, 84036 Germany. https://opac.hawlandshut.de/search?bvnr=BV047416805

7. Ostbayerische Technische Hochschule Amberg-Weiden, Hochschulbibliothek Amberg. Amberg/Oberpfalz, 92224 Germany. https://opac.othaw.de/TouchPoint/perma.do?q=+1035\%3D\%22BV047416805\%22+IN+\%5B2\%5D\&v=faw\&I=de

8. Universitätsbibliothek Bamberg. Bamberg, 96052 Germany.

9. Universitätsbibliothek München. München, 80539 Germany. https://opac.ub.unimuenchen.de/TouchPoint/perma.do?q=+0\%3D\%226064064\%22+IN+\%5B2\%5D\&v=sunrise\&I=de 
10. Bibliothek der Technischen Hochschule Aschaffenburg. THB Aschaffenburg. Aschaffenburg, 63743 Germany.

11. Hochschule Kempten, Hochschulbibliothek. Kempten, 87435 Germany. https://fketp20.bibbvb.de/TouchPoint/perma.do?q=+1035\%3D\%22BV047416805\%22+IN+\%5B2\%5D\&v=fke\&l=de

12. Universitätsbibliothek Kassel, Landesbibliothek und Murhardsche Bibliothek der Stadt Kassel. Kassel, 34127 Germany. https://hds.hebis.de/ubks/Record/HEB484063863

13. Justus-Liebig-Universität Gießen. Gießen, 35394 Germany.

Như vậy, thời gian để ấn phẩm này xuất hiện trong thư viện nhanh hơn rất nhiều so với cuốn The Vietnamese Social Sciences at a Fork in the Road [2] năm 2019. Để cuốn [2] có mặt trong thư viện đầu tiên (tức là có thể tìm được qua trang web của thư viện quốc tế), ước tính mất chừng 3-4 tháng. Cho tới hiện nay, có thể tìm được [2] tại hơn 40 thư viện, gần 2 năm sau khi phát hành. Trong khi đó, chỉ ngay trong tháng xuất bản, cuốn [1] đã có mặt tại hơn 10 thư viện đầu tiên, và dự kiến sẽ còn tiếp tục mở rộng hiện diện trong những tháng tới.

Về xuất xứ, [1] có liên quan mật thiết tới nghiên cứu "Alice in Suicideland: Exploring the Suicidal Ideation Mechanism through the Sense of Connectedness and Help-Seeking Behaviors" [3], cùng nhau chia sẻ tiếp cận BMF [4], phát triển dựa trên nền tảng [5-8].

\section{References}

[1] Vuong QH, Nguyen MH, Le TT. (2021). A Mindsponge-Based Investigation into the Psycho-Religious Mechanism Behind Suicide Attacks. De Gruyter.

[2] Vuong QH, Tran T. (2019). The Vietnamese Social Sciences at a Fork in the Road. De Gruyter.

[3] Nguyen MH, et al. (2021). Alice in Suicideland: Exploring the Suicidal Ideation Mechanism through the Sense of Connectedness and Help-Seeking Behaviors. International Journal of Environmental Research and Public Health, 18 (7), 3681.

[4] Nguyen MH, Le TT. (2021). Bayesian Mindsponge Framework. https://encyclopedia.pub/13852.

[5] Vuong QH. (2016). Global mindset as the integration of emerging socio-cultural values through mindsponge processes: A transition economy perspective. In: J. Kuada (ed.) Global Mindsets: Exploration and Perspectives (pp. 109-126). London: Routledge.

[6] Vuong QH, et al. (2020). Bayesian analysis for social data: A step-by-step protocol and interpretation. MethodsX, 7, 100924.

[7] Vuong QH, et al. (2020). Improving Bayesian statistics understanding in the age of Big Data with the bayesvl R package. Software Impacts, 4, 100016.

[8] La VP, Vuong QH. (2019). Package 'bayesvl': Visually Learning the Graphical Structure of Bayesian Networks and Performing MCMC with 'Stan'. The Comprehensive R Archive Network (CRAN). Retrieved from: https://osf.io/wyc6n 